\title{
Scientific seeds of inspiration
}

Our Journal Club articles explore historical scientific publications that have served as inspiration, be it for their originality, importance, interdisciplinary interest, elegance or surprising conclusions.

\section{Cf choose a research paper of any age ... and convey why the paper was influential}

Communicating and interpreting research findings is inherent to the process of scientific discovery and innovation. Global research output, and with it the number of scientific publications, continues to grow year on year. One of the unintended consequences of this increasing number of papers can be that researchers delve deeply into their individual research topics to the detriment of knowledge breadth. One could be forgiven to question why we, the editors of Nature Reviews Genetics, have chosen this point to implement a new article type on the journal. From our June 2021 issue onwards, we will begin to publish externally authored Journal Club articles that we hope will provide an insight into the many sources and avenues of scientific inspiration.

As editors, we appreciate how staying afloat in the deluge of novel research and data is not an easy feat. Since the journal first launched more than 20 years ago, it has regularly published Research Highlights, short articles written by the editors that bring interesting papers to the attention of our readers. Whereas Research Highlights focus on very recent research papers ( $<4$ weeks old), Journal Club articles will cover older, historical publications, with the intention of alerting our readers to articles that have a special significance to the field based on the personal perspective of the author.

Inspiration is often considered elusive, but many researchers can remember that lightbulb moment — while

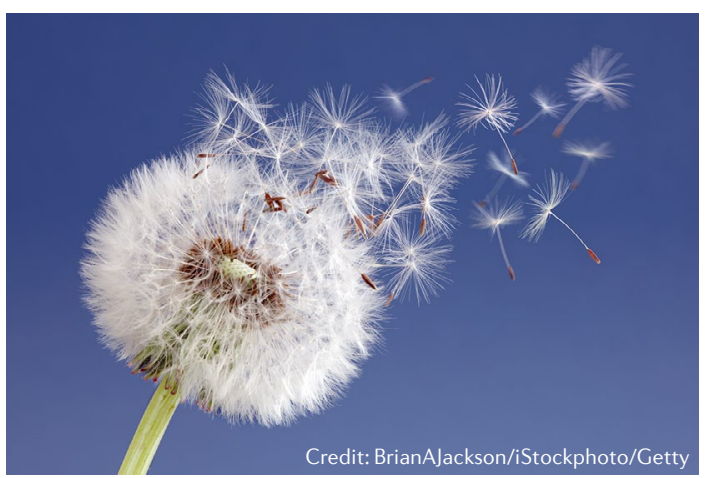

reading a paper with an unexpected result, a novel experimental approach or a groundbreaking technology when a new idea tentatively forms or a question emerges that (with the beauty of hindsight) will go on to influence future research. We think that the field can greatly benefit from hearing these stories and are therefore asking our contributors to choose a research paper of any age, relevant to our readership of geneticists or genomicists, and convey why the paper was influential and merits attention.

Indeed, by reflecting on a scientific publication that has driven the desire for a deeper understanding, and perhaps even influenced the path chosen from thereon in, we believe these accounts may prove inspirational themselves. For example, in our first Journal Club article, Neil Gemmell recalls pioneering work by Britten and Kohne, published in 1968, which ignited in him an enduring fascination as to why eukaryotic genomes contain substantial fractions of repetitive DNA ${ }^{1}$. In a second piece, Qifa Zhang describes how a 2008 publication in Nature Biotechnology on transgenic purple tomatoes inspired him to tackle food security by improving the nutrient and culinary value of black rice ${ }^{2}$.

The questions we ask authors to pose themselves when deciding on which paper to choose include: What paper inspired you to enter your field or pursue a particular course of work? What paper surprised you the most? What paper has most affected (or perhaps even revolutionized) your field? What paper would you recommend to every new starter in your lab? What is the most under-rated paper in your field?

We hope that reading about a publication from this personal viewpoint, and gaining insight into the trajectories it spawned or the research it led to, will provide you with your own seed of inspiration to nurture.

1. Gemmell, N. J. Repetitive DNA: genomic dark matter matters Nat. Rev. Genet. https://doi.org/10.1038/s41576-021-00354-8 (2021).

2. Zhang, Q. Purple tomatoes, black rice and food security Nat. Rev. Genet. https://doi.org/10.1038/s41576-021-00359-3 (2021). 